The Astrophysical Journal, 682:1256-1263, 2008 August 1

(C) 2008. The American Astronomical Society. All rights reserved. Printed in U.S.A.

\title{
HN Peg B: A TEST OF MODELS OF THE L TO T DWARF TRANSITION ${ }^{1}$
}

\author{
S. K. Leggett, ${ }^{2}$ D. Saumon, ${ }^{3}$ Loic Albert, ${ }^{4}$ Michael C. Cushing, ${ }^{5}$ Michael C. Liu, ${ }^{5,6}$ K. L. Luhman, \\ M. S. Marley, ${ }^{8}$ J. Davy Kirkpatrick, ${ }^{9}$ Thomas L. Roellig, ${ }^{8}$ and K. N. Allers ${ }^{5}$ \\ Received 2008 February 21; accepted 2008 April 8
}

\begin{abstract}
Luhman and collaborators recently discovered an early-T dwarf companion to the G0 dwarf star HN Peg, using Spitzer Infrared Array Camera (IRAC) images. Companionship was established on the basis of the common proper motion inferred from 1998 Two Micron All Sky Survey images and the 2004 IRAC images. In this paper we present new near-infrared imaging data which confirm the common proper motion of the system. We also present new 3-4 $\mu \mathrm{m}$ spectroscopy of HN Peg B, which provides tighter constraints on both the bolometric luminosity determination and the comparison to synthetic spectra. New adaptive optics imaging data are also presented, which show the T dwarf to be unresolved, providing limits on the multiplicity of the object. We use the age, distance, and luminosity of the solarmetallicity $\mathrm{T}$ dwarf to determine its effective temperature and gravity, and compare synthetic spectra with these values, and a range of grain properties and vertical mixing, to the observed $0.8-4.0 \mu \mathrm{m}$ spectra and mid-infrared photometry. We find that models with temperature and gravity appropriate for the older end of the age range of the system $(0.5 \mathrm{Gyr})$ can do a reasonable job of fitting the data, but only if the photospheric condensate cloud deck is thin, and if there is significant vertical mixing in the atmosphere. Dwarfs such as HN Peg B, with well-determined metallicity, radius, gravity, and temperature, will allow development of dynamical atmosphere models, leading to the solution of the puzzle of the L to T dwarf transition.
\end{abstract}

Subject headings: binaries: visual — stars: individual (2MASS J21442847+1446077, HN Peg, HN Peg B) — stars: low-mass, brown dwarfs

\section{INTRODUCTION}

Detailed studies of brown dwarf companions to main-sequence stars contribute significantly to our understanding of these fascinating objects (e.g., Gl 229B, Gl 570D, and HD 3651B; Marley et al. 1996; Geballe et al. 2001; Saumon et al. 2000, 2006a; Liu et al. 2007). This is because the primary star, if well studied, immediately gives us the brown dwarf's distance, chemical composition, and, most importantly (since brown dwarfs cool with time), constrains its age. Therefore the discovery of two $\mathrm{T}$ dwarfs with spectral types of T2.5 and T7.5 as companions to the nearby stars $\mathrm{HN}$ Peg (G0 V; $18.4 \mathrm{pc}$ ) and HD 3651 (K0 V; $11.1 \mathrm{pc})$, respectively, by Luhman et al. (2007, hereafter L07; HD 3651B was independently discovered by Mugrauer et al. [2006]) is an exciting and important result. Companionship of the T dwarfs was confirmed

\footnotetext{
1 Some of the data presented herein were obtained at the W. M. Keck Observatory, which is operated as a scientific partnership among the California Institute of Technology, the University of California, and the National Aeronautics and Space Administration. The Observatory was made possible by the generous financial support of the W. M. Keck Foundation. Some data were also obtained at the Canada-France-Hawaii Telescope (CFHT), which is operated by the National Research Council of Canada, the Institut National des Sciences de l'Univers of the Centre National de la Recherche Scientifique of France, and the University of Hawaii.

${ }^{2}$ Gemini Observatory, Northern Operations Center, 670 North A'ohoku Place, Hilo, HI 96720.

${ }^{3}$ Los Alamos National Laboratory, P.O. Box 1663, MS F663, Los Alamos, NM 87545.

4 Canada-France-Hawaii Telescope Corporation, 65-1238 Mamalahoa Highway, Kamuela, HI 96743.

5 Institute for Astronomy, University of Hawaii, 2680 Woodlawn Drive, Honolulu, HI 96822.

6 Alfred P. Sloan Research Fellow.

7 Department of Astronomy and Astrophysics, Pennsylvania State University, University Park, PA 16802.

${ }^{8}$ NASA Ames Research Center, Mail Stop 245-3, Moffett Field, CA 94035.

9 IPAC, California Institute of Technology, Mail Code 100-22, 770 South Wilson Avenue, Pasadena, CA 91125.
}

using proper motions determined from Two Micron All Sky Survey (2MASS; Skrutskie et al. 2006) and Spitzer Space Telescope (Werner et al. 2004) Infrared Array Camera (IRAC; Fazio et al. 2004) images separated by roughly 6 years.

In this paper we present new observational data for one of the L07 T dwarfs, the proposed companion to HN Peg, HN Peg B (or 2MASS J21442847+1446077). The G0 V primary, HN Peg (HD 206860), is a BY Draconis variable, where the variability has been interpreted as due to spots on the surface, and the 24.9 day period as the rotation period of the star (Blanco et al. 1979). It is relatively young, with an age of $0.3 \pm 0.2 \mathrm{Gyr}$ (L07; see also $\S 5$ below), and has a debris disk with a radius determined from the $70 \mu \mathrm{m}$ flux excess of $\sim 7 \mathrm{AU}$ ( Trilling et al. 2008). The proposed companion is $43.2^{\prime \prime}$ away from the primary, which translates to $795 \mathrm{AU}$ at the distance of the primary. Recent multiplicity studies suggest that the frequency of brown dwarf companions at wide separations is low (e.g., Kraus et al. 2008); however, the statistical significance of these findings is not well constrained due to the small sample sizes. This system adds one more wideseparation $\mathrm{G}$ star and brown dwarf pair to such studies.

Here we use the new observational data to confirm that the early-T dwarf is indeed a companion to HN Peg, to investigate its multiplicity, and to refine the determination of its luminosity. The luminosity allows us to constrain the temperature and gravity of the dwarf from the known age and distance of the solarmetallicity system. Having determined the temperature and gravity, we compare the red, near-infrared, and 3-4 $\mu \mathrm{m}$ spectra, as well as the IRAC $4-8 \mu \mathrm{m}$ photometry, to synthetic spectra and photometry, and present the results of the model comparisons. We find that the models can reproduce the data quite well, and hence bring us closer to resolving the puzzle of this poorly understood phase of brown dwarf evolution: the transition from the dusty, red in the near-infrared, L dwarfs, to the clear atmosphere, blue in the near-infrared, T dwarfs (e.g., Burgasser et al. 2002; Knapp et al. 2004). 


\section{NEW OBSERVATIONS}

\subsection{Near-Infrared Imaging}

\subsubsection{WIRCam Imaging}

$J$-band imaging was conducted with WIRCam at the CanadaFrance-Hawaii Telescope (CFHT; Puget et al. 2004) at three different epochs (2006 September 13, 2007 May 7, and 2007 July 12 [UT]) using a 9-point dithering pattern of $60^{\prime \prime}$ amplitude. HN Peg was positioned in the corner of the northeast detector, about $85^{\prime \prime}$ off the center of the mosaic. The total integration time was 9 minutes for the first two epochs and 17 minutes for the last. Images were preprocessed and sky subtracted at CFHT with the I'iwi pipeline ${ }^{10}$ and median stacked using the Terapix software suite (sextractor, Bertin \& Arnouts 1996; scamp, Bertin 2006; and swarp ${ }^{11}$ ). The internal astrometry at each epoch is better than 40 mas rms, and the image quality on the resulting stacks is $0.76^{\prime \prime}, 1.02^{\prime \prime}$, and $0.81^{\prime \prime}$ for the three respective epochs. Using the 2MASS pointsource catalog to fix the astrometry of each reduced image implies rms errors of $0.25^{\prime \prime}$, and this is the uncertainty that we adopt for the coordinates of HN Peg B derived from these data.

\subsubsection{SOFI Imaging}

Through a search of the data archive for the European Southern Observatory, we found publicly available images of HN Peg that were obtained with the SOFI near-infrared camera on the $3.5 \mathrm{~m}$ New Technology Telescope at La Silla Observatory. These data were collected on the night of 2006 June 15 through program 077.C-0704. The instrument contained one $1024 \times 1024$ $\mathrm{HgCdTe}$ Hawaii array with a plate scale of 0.288 arcsec pixel $^{-1}$. Ten dithered $60 \mathrm{~s}$ exposures of HN Peg were obtained through an $H$-band filter. After these images were flat-fielded, registered, and combined, the resulting image exhibited a full width at halfmaximum (FWHM) near $1^{\prime \prime}$ for point sources. We determined the plate solution of the combined image using coordinates measured by 2MASS for sources which were well detected but unsaturated, and not obviously multiple. The rms errors in the astrometry is $0.19^{\prime \prime}$.

\subsubsection{NSFCAM2 Imaging}

$K$-band images were obtained of HN Peg B on 2007 September 27 (UT) using NSFCAM2 (Shure et al. 1994) at the NASA Infrared Telescope Facility (IRTF). Twelve $60 \mathrm{~s}$ dithered frames were obtained, on a nonphotometric night, with seeing around FWHM $0.8^{\prime \prime}$ at $K$. The camera field of view is $80^{\prime \prime} \times 80^{\prime \prime}$, and the pixel scale is 0.04 arcsec pixel $^{-1}$. Three well-detected stars with 2MASS near-infrared magnitudes of 14-15 (i.e., not HN Peg or HN Peg B) were included in five of these frames, and these were used to define the astrometry. The uncertainty in these coordinates is estimated to be $0.1^{\prime \prime}$ from the standard deviation of the values derived from the five frames, and is similar to the rms error in the astrometric calibration of each frame.

\section{2. $3.5 \mu \mathrm{m}$ Spectroscopy}

We acquired spectra from 2.96 to $4.07 \mu \mathrm{m}$ of HN Peg B using the Near-InfraRed Imager and spectrograph (NIRI; Hodapp et al. 2003 ) on the Gemini North Telescope. The 3-4 $\mu$ m wavelength region includes both the $\nu_{3}$ fundamental absorption band of $\mathrm{CH}_{4}$ at $3.3 \mu \mathrm{m}$ and a bright flux peak around $4 \mu \mathrm{m}$. This spectral region is therefore useful for defining the overall shape of the spectral energy distribution and for measuring the bolometric luminosity.

\footnotetext{
${ }^{10}$ See http://www.cfht.hawaii.edu/Instruments/Imaging/WIRCam/.

11 See http://terapix.iap.fr.
}

Over the course of four nights (2007 July 26 and 30; August 1 and 5 [UT]) we obtained a total of $4.67 \mathrm{hr}$ of data, consisting of 280, $60 \mathrm{~s}$ exposures, each made up of 30 co-adds of $2 \mathrm{~s}$ integrations (short integration times are necessary due to the high and variable $L$-band background). The $L$-grism was used with the $L$ order-sorting filter. The nights were required to be photometric with good image quality, to maximize the flux through NIRI's 6 pixel $\left(0.72^{\prime \prime}\right)$ slit. The spectral resolving power provided by this configuration is $R \equiv \lambda / \Delta \lambda \approx 460$.

The F0 V star HD 194822 or the A1 V star HD 217186 were used as calibrators. The science target and calibrators were observed using $\pm 3^{\prime \prime}$ offsets along the slit in an ABBA pattern. Flat fields were obtained using the Gemini calibration unit, and bad pixel masks were derived from dark frames. Wavelength calibration was achieved using telluric and intrinsic spectral features in the calibration stars. IRAF routines were used to mask bad pixels, flat field each frame, subtract pairs of frames, and produce one to five stacked images for each night, each of which contained a positive and negative spectrum and represented 2040 minutes of observation. Figaro routines were used to extract the spectra, wavelength calibrate, and flux calibrate using the telluric standards. The final absolute flux calibration was achieved using the Spitzer IRAC $3.6 \mu \mathrm{m}$ photometry given by L07 using the technique given in Cushing et al. (2006). Finally the spectrum was rebinned so that each pixel corresponds to a single resolution element. The signal-to-noise ratio ranges from 5 at the blue end of the spectrum (where there is little flux) to $15-20$, at the brighter red end. The spectrum is presented later, in $\S 4$.

\subsection{Keck Laser Guide Star Adaptive Optics Imaging}

The G0 primary, HN Peg, has been the target of radial velocity monitoring, but there is no evidence of planets orbiting the star (Fischer \& Valenti 2005; König et al. 2006). Hence the system seems to be composed of the star and its brown dwarf companion, with a separation of $795 \mathrm{AU}$.

We searched for companions to HN Peg B on 2006 October 14 using the laser guide star adaptive optics (LGS AO) system (van Dam et al. 2006; Wizinowich et al. 2006) of the 10 m Keck II Telescope on Mauna Kea, Hawaii. Conditions were photometric with variable seeing. We used the facility infrared camera NIRC2 with its narrow field-of-view camera, which produces an image scale of $9.963 \pm 0.011$ mas pixel $^{-1}$ (Pravdo et al. 2006) and a $10.2^{\prime \prime} \times 10.2^{\prime \prime}$ field of view. The LGS provided the wave front reference source (equivalent to a $V \approx 9.6$ mag star) for $\mathrm{AO}$ correction, with the exception of tip-tilt motion. Tip-tilt aberrations and quasi-static changes in the image of the LGS were measured contemporaneously with a lower bandwidth wave front sensor monitoring the $R=12.7 \mathrm{mag}$ field star USNO-B1.0 214428+ 14465 (Monet et al. 2003), located 44" away from HN Peg B. Technical difficulties with this second wave front sensor led to somewhat degraded image quality in the inner $\approx 0.3^{\prime \prime}$ radius compared to typical data.

We obtained a series of dithered images, offsetting the telescope by a few arcseconds, with a total integration time of $420 \mathrm{~s}$. We used the $K$-band filter from the Mauna Kea Observatories (MKO) filter consortium (Simons \& Tokunaga 2002; Tokunaga et al. 2002). The images were reduced in a standard fashion. We constructed flat fields from the differences of images of the telescope dome interior with and without continuum lamp illumination. Master sky frames were created from the median average of the bias-subtracted, flat-fielded images and subtracted from the individual images. Images were registered and stacked to form a final mosaic, with a FWHM of $0.06^{\prime \prime}$ and a Strehl ratio of 


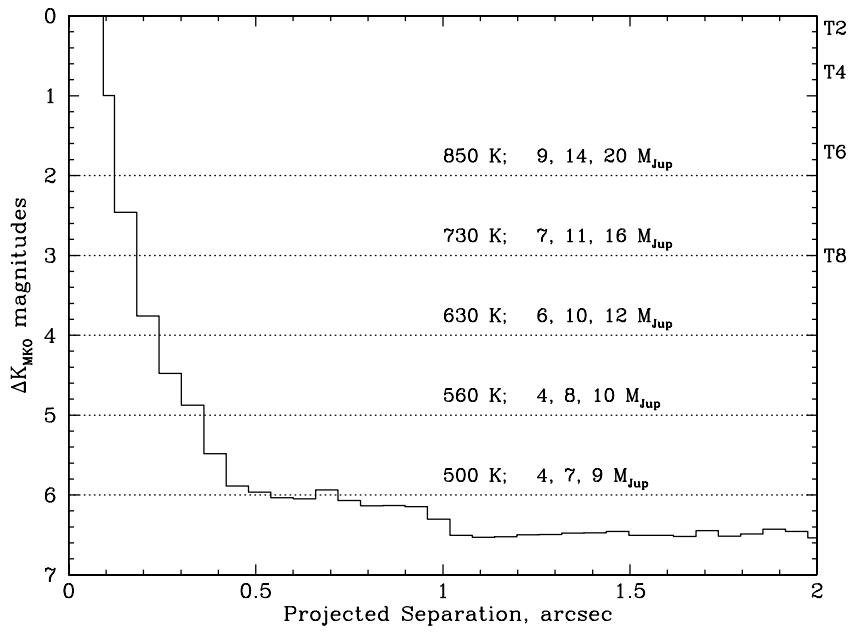

FIG. 1.- Limits on multiplicity of HN Peg B, derived from our AO imaging. Spectral types corresponding to the $K$ magnitude of a companion to HN Peg B are shown along the right axis, and effective temperatures and masses of any companion are shown along the dotted lines, for ages $0.1,0.3$, and $0.5 \mathrm{Gyr}$.

0.34 . No companions were clearly detected in a $6^{\prime \prime} \times 6^{\prime \prime}$ region, corresponding to $110 \mathrm{AU}$, centered on HN Peg B.

We determined limits on any companions by first convolving the final mosaic with an analytical representation of the pointspread function's (PSF's) radial profile, modeled as the sum of multiple Gaussians. We then measured the standard deviation in concentric annuli centered on HN Peg B, normalized by the peak flux of the targets, and took $10 \sigma$ as the flux ratio limits for any companions. These limits were verified with implantation of fake companions into the image using translated and scaled versions of the science target. Inside about $0.25^{\prime \prime}$ in radius, the complex structure of the PSF is the main noise source. From about $0.25^{\prime \prime}$ to $1.0^{\prime \prime}$, the noise primarily arises from shot noise of the PSF halo and at larger separations from both shot noise of the sky emission and detector read noise.

Figure 1 presents the companion detection limits. We use the Liu et al. (2006) polynomial fits for $K$-band absolute magnitude as a function of spectral type to convert the brightness limits into spectral types for field ultracool dwarfs. Evolutionary models calculated by one of us (D. S.), using cloud-free atmospheres, were used to convert the $K$ limits into $T_{\text {eff }}$ and mass, given the age range of 0.1-0.5 Gyr for the HN Peg system. The observations show that there is no companion warmer than $\sim 500 \mathrm{~K}$ or more massive than $\sim 10 M_{\text {Jupiter }}$ at $0.3^{\prime \prime}-6^{\prime \prime}$, or $5.5-110$ AU from HN Peg B. The luminosity of the system rules out the presence a similar-mass companion ( $\S \S 5$ and 6), i.e., a companion with mass $>10-20 M_{\text {Jupiter }}$ or $T_{\text {eff }} \gtrsim 1000 \mathrm{~K}$. Thus any companion to
HN Peg B must be less massive than $\sim 10 M_{\text {Jupiter }}$ and cooler than $500-700 \mathrm{~K}$ (depending on the exact age of the system).

Recent high spatial resolution imaging of brown dwarfs has found that the binary frequency among T2-T4.5 dwarfs is unusually high, with most early-T dwarfs consisting of a similar mass, but very different color, pair, made up of a late-L or very early-T and a mid- to late-T dwarf (e.g., Burgasser et al. 2006b; Liu et al. 2006). This finding is appealing, as it reduces the $J$-band brightening seen for early- $\mathrm{T}$ dwarfs, making the $\mathrm{L}$ to $\mathrm{T}$ transition easier to model. HN Peg B appears to be one of the rare T2.5 dwarfs which is not composed of a similar-mass pair of dwarfs.

\section{ASTROMETRY OF THE HN Peg SYSTEM}

\subsection{Proper-Motion Determination}

We used the WIRCam, SOFI, and NSFCAM2 imaging data described above, as well as the Spitzer IRAC images obtained for the L07 program on 2004 July 10, to determine accurate coordinates for the proposed companion to $\mathrm{HN}$ Peg at multiple epochs. The results are given in Table 1. In all cases the dwarf was well detected, and 2MASS stars were used to refine the coordinate systems.

In the case of the IRAC data, coordinates could be determined from the four IRAC channels to $0.1^{\prime \prime}-0.22^{\prime \prime}$ in right ascension and $0.1^{\prime \prime}-0.14^{\prime \prime}$ in declination. The uncertainty in these coordinates is estimated from the standard deviation of the values derived from the four frames, and is similar to the rms error in the astrometric calibration of each frame.

Figure 2 shows the difference between the 2004, 2006, and 2007 epoch measurements of the right ascension and declination of the dwarf, and the values that it would have were it a companion to HN Peg. The 1998 2MASS coordinates provide the initial values. We adopt a proper motion for $\mathrm{HN}$ Peg of $231.2 \pm 1.0$ mas yr $^{-1}$

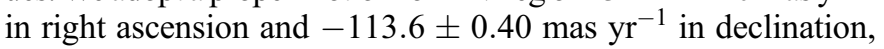
based on a weighted average of the Hipparcos (Perryman et al. 1997) and PPM North (Roeser \& Bastian 1988) catalogs. The errors in the values plotted are the combined uncertainties in our astrometric measurements, the 2MASS reference system, and the proper motion of HN Peg.

The NSFCAM 2 astrometry differs in right ascension by $\sim 0.3^{\prime \prime}$ from the WIRCam results, and also disagrees with other available data. The NSFCAM2 field is known to suffer from distortion at the $0.1^{\prime \prime}$ level. ${ }^{12}$ It appears that this distortion, combined with the small number of stars used to define the astrometry, leads us to underestimate the error on this measurement.

The proper motion of the T dwarf in declination is small and is consistent with that of $\mathrm{HN}$ Peg along this axis. While the

12 See http://irtfweb.ifa.hawaii.edu/ nsfcam2/Distortion\%20Analysis.html.

TABLE 1

Astrometry For HN Peg B (2MASS J21442847+1446077)

\begin{tabular}{|c|c|c|c|c|c|}
\hline Epoch Year & $\begin{array}{l}\text { Right Ascension } \\
\quad(\mathrm{J} 2000.0)\end{array}$ & $\begin{array}{l}\sigma(\text { R.A. }) \\
(\operatorname{arcsec})\end{array}$ & $\begin{array}{l}\text { Declination } \\
(\mathrm{J} 2000.0)\end{array}$ & $\begin{array}{l}\sigma(\text { Decl. }) \\
(\operatorname{arcsec})\end{array}$ & Survey or Instrument \\
\hline $1998.734 \ldots$. & 214428.472 & 0.07 & +144607.80 & 0.07 & 2MASS \\
\hline $2004.441 \ldots \ldots \ldots$ & 214428.548 & 0.22 & +144607.06 & 0.14 & IRAC \\
\hline 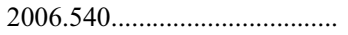 & 214428.595 & 0.19 & +144606.86 & 0.19 & SOFI \\
\hline 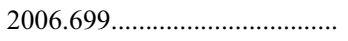 & 214428.582 & 0.25 & +144606.81 & 0.25 & WIRCam \\
\hline 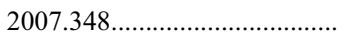 & 214428.601 & 0.25 & +144606.73 & 0.25 & WIRCam \\
\hline 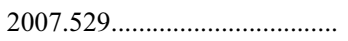 & 214428.599 & 0.25 & +144606.73 & 0.25 & WIRCam \\
\hline 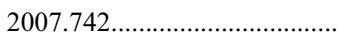 & 214428.581 & 0.10 & +144606.79 & 0.10 & NSFCAM2 \\
\hline
\end{tabular}

NoтE.-Units of right ascension are hours, minutes, and seconds, and units of declination are degrees, arcminutes, and arcseconds. 


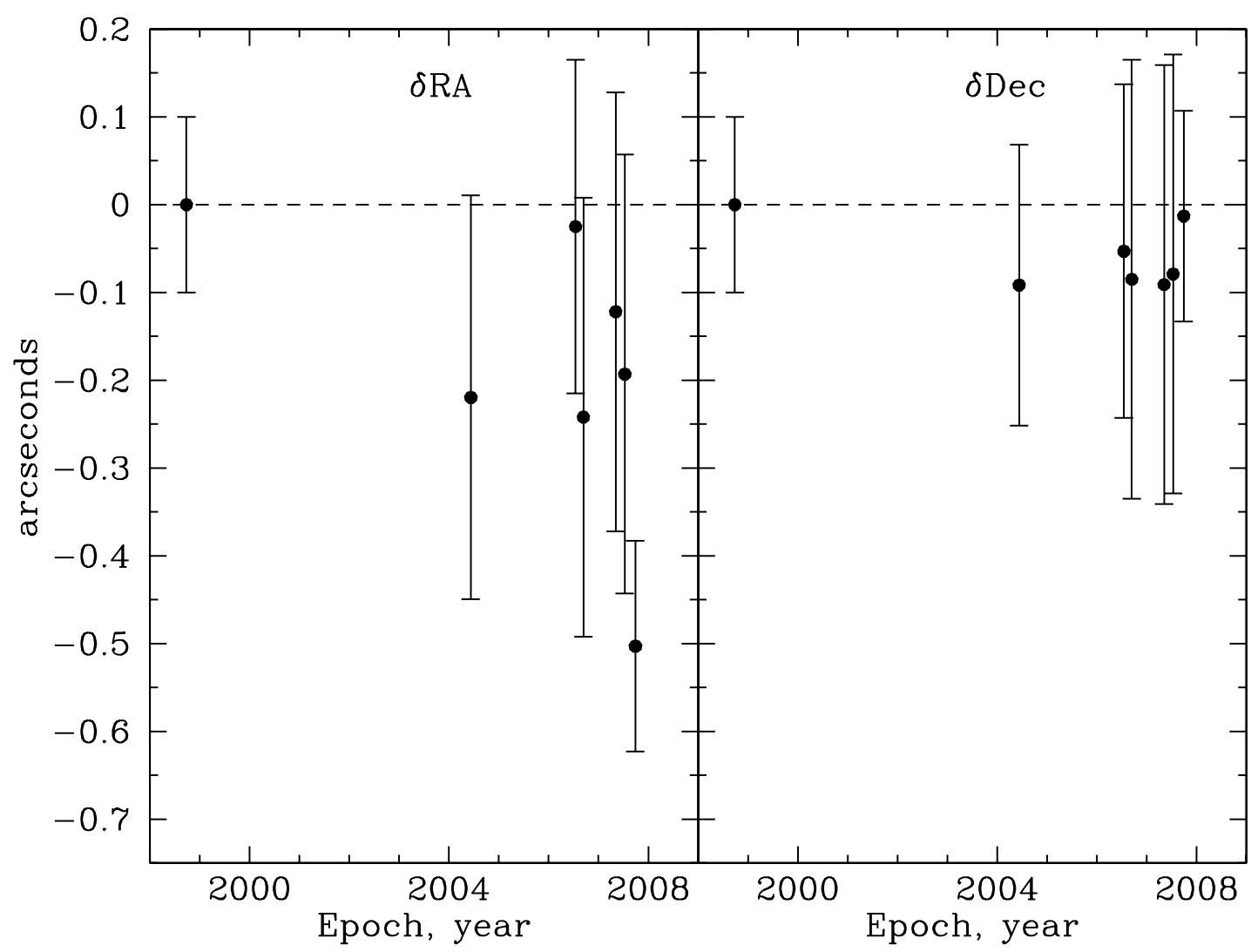

FIG. 2.-Difference between the positions of the proposed companion to HN Peg at various epochs, and its $1998.72 \mathrm{MASS}$ position, updated assuming common proper

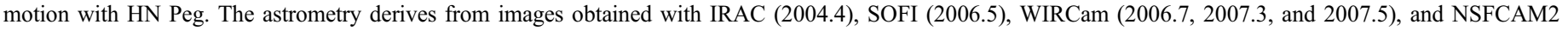
(2007.7).

agreement in right ascension is not as good as that in declination, the values are consistent with companionship within the measurement errors, with the exception of the NSFCAM2 value. Given the low probability of detecting a field $\mathrm{T}$ dwarf (see the following section), we interpret this level of agreement as confirmation of companionship.

\subsection{Probability of Detecting a Background T Dwarf}

The probability of L07 discovering a background T dwarf near HN Peg is low, although finite. Metchev et al. (2008) determine the space density of T0-T8 dwarfs to be $7.2 \times 10^{-3} \mathrm{pc}^{-3}$. The L07 study required a good detection in all four IRAC bands, limiting the detection of early-T dwarfs to around $30 \mathrm{pc}$ (and later $\mathrm{T}$ dwarfs to smaller distances). The number of $\mathrm{T}$ dwarfs within a $30 \mathrm{pc}$ volume is 810 , using the Metchev et al. density value. The field of view of IRAC is $5.2^{\prime}$, and thus each star observed by L07 samples $27.04 \operatorname{arcmin}^{2}$ or $1.82 \times 10^{-7}$ of the celestial sphere. The L07 sample size is 121 targets, so we would expect to find $<0.018$ field $\mathrm{T}$ dwarfs in this survey, as the $30 \mathrm{pc}$ distance is an upper limit. Assuming Poisson statistics, the probability of detecting one field $\mathrm{T}$ dwarf given an expectation rate of $<0.018$ is $<2 \%$. However, the probability of finding such a field $\mathrm{T}$ dwarf with a similar proper motion to HN Peg (as described in the previous section) is negligibly small.

\section{THE SPECTRAL ENERGY DISTRIBUTION AND LUMINOSITY OF HN Peg B}

L07 determined the spectral type of HN Peg B to be T2.5 \pm 0.5 by comparing its near-infrared spectrum to the $\mathrm{T}$ dwarf spectral templates presented by Burgasser et al. (2006a). Using the L07 spectrum, we computed the values of the spectral indices defined in Burgasser et al. and find spectral types ranging from $\mathrm{T} 2$ to $\mathrm{T} 4.5$, with an average of $\mathrm{T} 3 \pm 1$ (a significant range in type is not uncommon for $\mathrm{L}$ to $\mathrm{T}$ transition dwarfs; see, for example, Table 9 in Knapp et al. [2004]). This spectral type is in good agreement with the spectral type derived by the direct comparison technique.

Figure 3 shows the $0.8-4.1 \mu \mathrm{m}$ spectrum of the Burgasser et al. (2006a) T2 spectral standard SDSS J125453.90-012247.4 (hereafter SDSS 1254-0122) from Cushing et al. (2005), and that for HN Peg B. The spectra have been scaled to demonstrate the

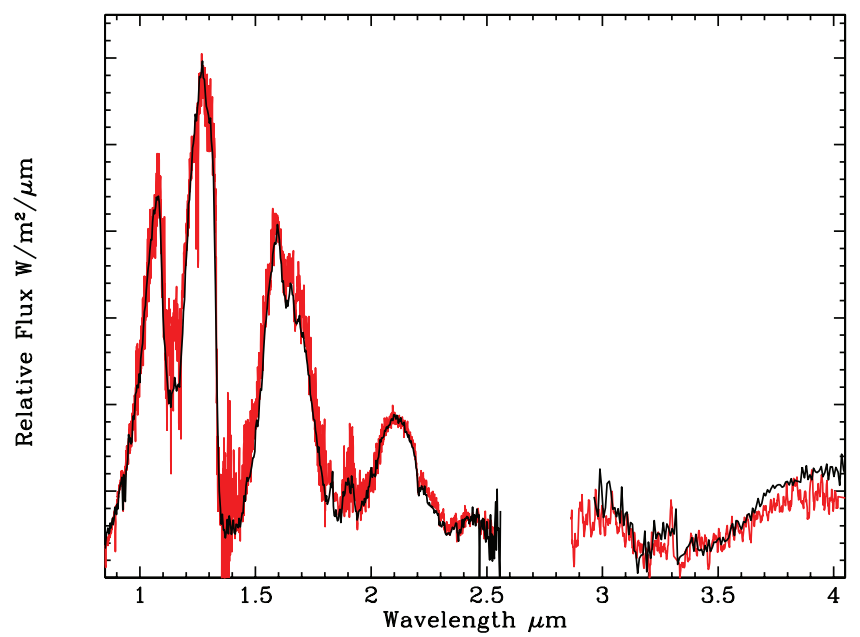

FIG. 3.-Observed spectra for the T2 template SDSS J1254-0122 (red; Cushing et al. 2005) and HN Peg B (black; L07 and this work). 
TABLE 2

Luminosity-Constrained Parameters for HN Peg B

\begin{tabular}{ccccc}
\hline \hline $\begin{array}{c}\text { Age } \\
(\mathrm{Gyr})\end{array}$ & $\begin{array}{c}T_{\text {eff }} \\
(\mathrm{K})\end{array}$ & $\log g$ & $\begin{array}{c}\text { Radius } \\
\left(R_{\odot}\right)\end{array}$ & $\begin{array}{c}\text { Mass } \\
\left(M_{\mathrm{J}}\right)\end{array}$ \\
\hline $0.1 \ldots \ldots \ldots \ldots \ldots$. & 1015 & 4.22 & 0.134 & 12 \\
$0.5 \ldots \ldots \ldots \ldots \ldots$. & 1115 & 4.81 & 0.101 & 28 \\
\hline
\end{tabular}

similarity between their spectral energy distributions (SEDs). Comparing the scaling factor to that derived from the trigonometric parallaxes measured for SDSS 1254-0122 and HN Peg (Dahn et al. 2002; Perryman et al. 1997) shows that SDSS 12540122 is brighter than HN Peg B by a factor of 1.55 . It has been suggested that SDSS 1254-0122 is multiple, based on its absolute magnitude (Burgasser et al. 2006b; Liu et al. 2006), although it remains unresolved by the Hubble Space Telescope (Burgasser et al. 2006b). The fact that HN Peg B is not brighter than SDSS 1254-0122 supports the conclusion derived from the AO imaging of the dwarf, that HN Peg B does not have a close companion with similar mass. Compared to the T2 dwarf SDSS 1254-0122, HN Peg B has slightly stronger $\mathrm{H}_{2} \mathrm{O}$ absorption at $1.1 \mu \mathrm{m}$, slightly stronger $\mathrm{CH}_{4}$ at $1.6 \mu \mathrm{m}$, and is brighter at $4 \mu \mathrm{m}$, supporting the slightly later type of $\mathrm{T} 2.5-\mathrm{T} 3$.

We have determined the bolometric flux at Earth of HN Peg B by integrating the observed $0.8-4 \mu \mathrm{m}$ spectrum and adding the contribution of longer wavelengths using a synthetic spectrum. Initially we chose a synthetic spectrum with $T_{\text {eff }}=1400 \mathrm{~K}$, the temperature of SDSS 1254-0122 (Golimowski et al. 2004), due to the similarity in the SED. The model flux was scaled by the observed IRAC fluxes of HN Peg B. After deriving the luminosity in this way, evolutionary models were used to constrain the $T_{\text {eff }}$ and $\log g$ (see the following section), and the luminosity was rederived using the long-wavelength flux of a cooler $T_{\text {eff }}=$ $1115 \mathrm{~K}$ model, as indicated by the evolutionary model. The difference in the measured luminosity is $3.5 \%$, less than the uncertainty in the measurement, and the change in the $T_{\text {eff }}$ derived from the luminosity would be $<5 \mathrm{~K}$; we have not rederived the $T_{\text {eff }}$ and $\log g$.

The value derived for the luminosity at the Earth, using the cooler model long-wavelength extension, is $1.66 \times 10^{-15} \mathrm{~W} \mathrm{~m}^{-2}$, with an estimated uncertainty of $5 \%$. Adopting the HN Peg distance of $18.4 \pm 0.2 \mathrm{pc}$ (Perryman et al. 1997) implies a luminosity given by $\log L / L_{\odot}=-4.76 \pm 0.02$, in agreement with the value found by $\mathrm{L} 07$ of $-4.77 \pm 0.03$.

\section{AGE, METALLICITY, TEMPERATURE, AND GRAVITY OF HN Peg B}

L07 consider the Li, rotational, and chromospheric properties of $\mathrm{HN}$ Peg A to determine an age for the HN Peg system of $0.3 \pm$ 0.2 Gyr. This agrees with the recently derived gyrochronology age of $0.24 \pm 0.03 \mathrm{Gyr}$ (Barnes 2007). The system has solar metallicity: Valenti \& Fischer $(2005)$ report $[\mathrm{M} / \mathrm{H}]=-0.01 \pm 0.03$ for HN Peg A.

Evolutionary models calculated by one of us (D. S.) show that a T dwarf aged 0.1-0.5 Gyr, with our measured luminosity, has $T_{\text {eff }} \mathrm{K} ; \log g$; radius $R / R_{\odot}$; and mass of $1015 \mathrm{~K}, 4.22,0.134$, and $12 M_{\text {Jupiter }}$ at the younger age; and mass of $1115 \mathrm{~K}, 4.81,0.101$, and $28 M_{\text {Jupiter }}$ at the older age. These values are summarized in Table 2, and are consistent with the Burrows et al. (1997) and Baraffe et al. (2003) evolutionary sequences shown in Figures 10 and 11 of L07, although the temperature is slightly lower than that adopted by $\mathrm{L} 07$ of $1130 \mathrm{~K}$.
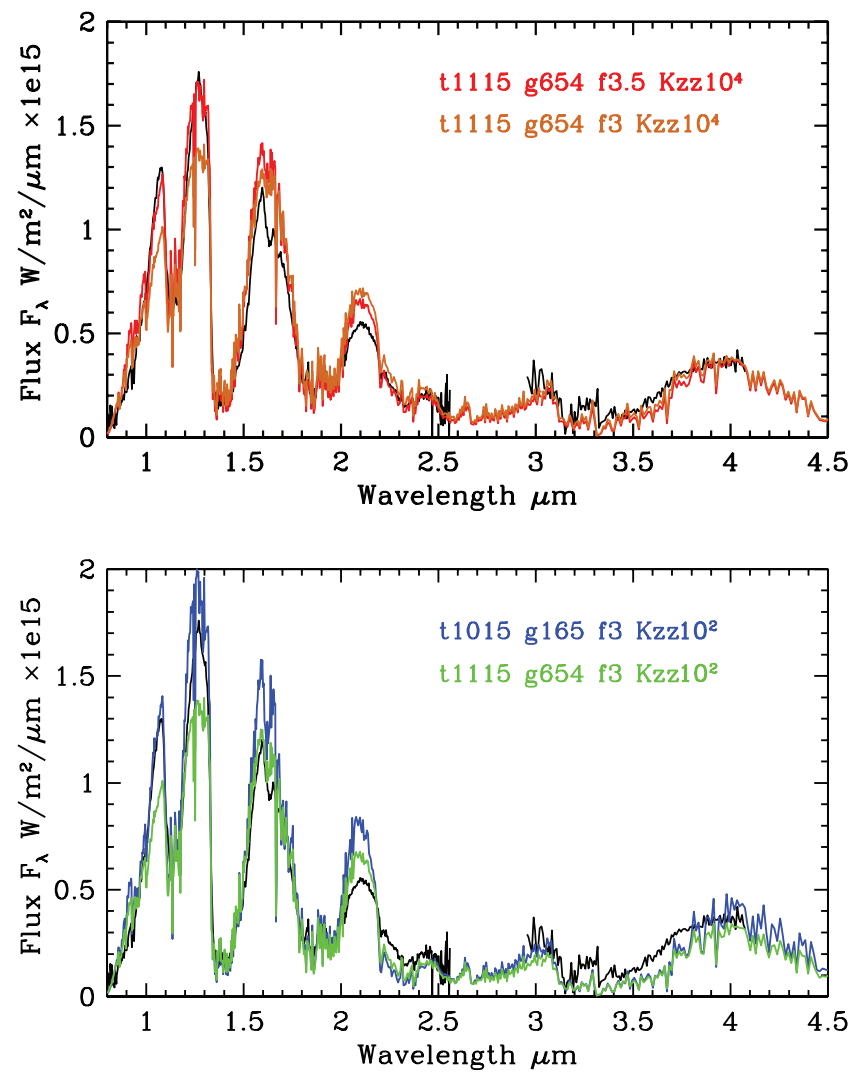

FIG. 4.- Observed spectrum of HN Peg B (heavy black line), compared to synthetic spectra with various $T_{\text {eff }}$, gravity, sedimentation efficiency $f_{\text {sed }}$, and vertical mixing diffusion coefficient $K_{z z}$ parameters, as indicated in the legends. The model fluxes have been scaled to flux at the Earth using the known distance to HN Peg and the HN Peg B radii calculated by evolutionary models. The top panel shows our best-fitting model (red line) together with a similar model with thicker cloud decks (brown line). The bottom panel shows the best-fitting cooler model (blue line), together with a warmer model spectrum demonstrating the effect of less vertical transport (green line; compare to brown line in top panel).

As also noted in $\mathrm{L} 07$, a temperature of $\sim 1065 \mathrm{~K}$ is significantly cooler than that of the typical field $\mathrm{T} 2-\mathrm{T} 3$ dwarf, which has $T_{\text {eff }} \sim 1200-1400 \mathrm{~K}$ (Golimowski et al. 2004). Cushing et al. (2008) determine $T_{\text {eff }}=1200-1400 \mathrm{~K}$ for the very spectrally similar T2 reference dwarf SDSS J1254-0122 (Fig. 3), from model analyses of the observed spectra, in agreement with the value determined from luminosity arguments by Golimowski et al. (2004) of $1425 \pm 175 \mathrm{~K}$. However, for HN Peg B to be as hot as $1200 \mathrm{~K}$, its age would need to be $\sim 1 \mathrm{Gyr}$, in violation of the $\mathrm{Li}$, rotation, and chromospheric activity constraints (L07). The young L7.5 dwarf HD 203030B (Metchev \& Hillenbrand 2006) also has an apparently low temperature (of $1200 \mathrm{~K}$ ), and it has been suggested that the temperature of the L to $\mathrm{T}$ dwarf transition may be gravity dependent. Benchmark transition dwarfs such as HN Peg B, with well-determined age and metallicity, are clearly important for studies of the properties that control the $\mathrm{L}$ to $\mathrm{T}$ dwarf transition.

In the following section we explore the fits of synthetic spectra and photometry to the HN Peg B data, and investigate how well our current atmospheric models can do, given the tight constraints on temperature, gravity, and radius that the evolutionary sequences provide. Note that if HN Peg B consists of an unresolved pair of identical dwarfs, then the luminosity is halved and $T_{\text {eff }}$ becomes $\sim 900 \mathrm{~K}$, for the age of the system. The comparison to the spectra below shows that our models do not support such a low temperature, consistent with the dwarf being single, or being 

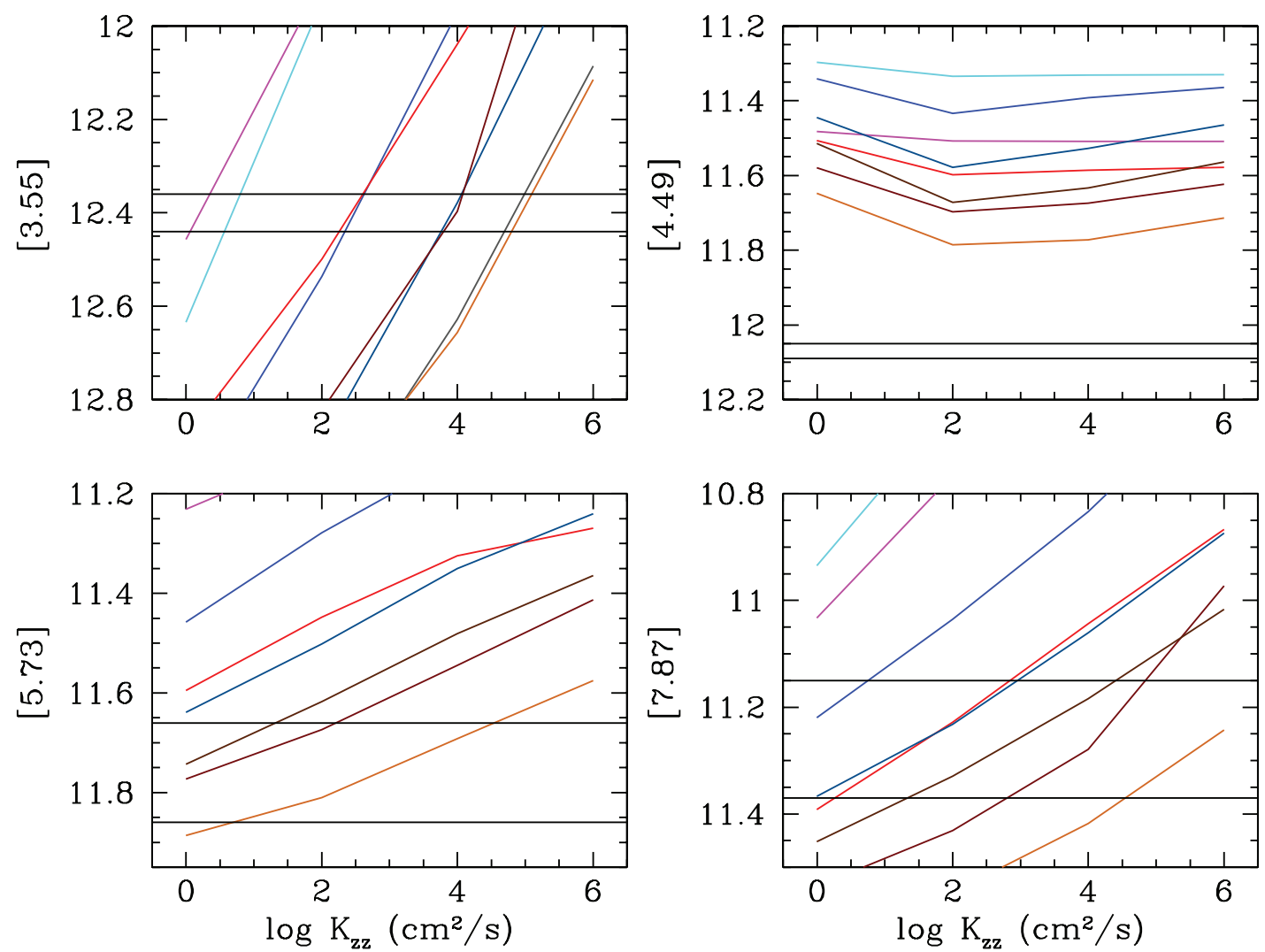

FIG. 5.- Absolute IRAC magnitudes for HN Peg B (range indicated by horizontal black lines), compared to synthetic photometry from our models as a function of vertical

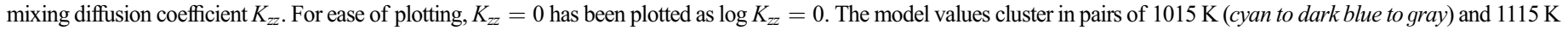
( pink to dark red to brown), with sedimentation parameter $f_{\text {sed }}=1,2,3$, and 4 from left to right for the [3.55] panel, and from top to bottom in the other panels.

composed of a significantly unequal-mass system, in agreement with the conclusions reached in $\S 2.3$ above.

\section{MODEL COMPARISONS}

The model atmospheres used here self-consistently include the formation of condensate clouds (Marley et al. 2002). The parameter $f_{\text {sed }}$ is a measure of the efficiency of condensate sedimentation relative to turbulent mixing (Ackerman \& Marley 2001). Larger values of $f_{\text {sed }}$ imply larger particle sizes, greater sedimentation efficiency, and thus thinner condensate clouds. Generally, $f_{\text {sed }}=2$ reproduces the colors and spectra of $\mathrm{L}$ dwarfs well, and $f_{\text {sed }}=4$ or cloud-free models reproduce the colors and spectra of later T dwarfs well (e.g., Knapp et al. 2004; Cushing et al. 2008).

The models also include vertical transport in the atmosphere, which affects the chemical abundance of species involving $\mathrm{C}, \mathrm{N}$, and $\mathrm{O}$. The extremely stable $\mathrm{CO}$ and $\mathrm{N}_{2}$ molecules can be dredged from deep layers into the photosphere, enhancing the abundances of these species, and decreasing the abundance of $\mathrm{CH}_{4}, \mathrm{H}_{2} \mathrm{O}$, and $\mathrm{NH}_{3}$ (e.g., Fegley \& Lodders 1996; Saumon et al. 2003, 2006b; Hubeny \& Burrows 2007). This mechanism is parameterized in our models by a diffusion coefficient $K_{z z} \mathrm{~cm}^{2} \mathrm{~s}^{-1}$. The larger the $K_{z z}$, the greater the enhancement of $\mathrm{CO}$ and $\mathrm{N}_{2}$ over $\mathrm{CH}_{4}$ and $\mathrm{NH}_{3}$. For T dwarfs, the effect is significant in the mid-infrared, where the $3 \mu \mathrm{m} \mathrm{CH}_{4}$ absorption band is weakened, the $4.5 \mu \mathrm{m}$ $\mathrm{CO}$ absorption band is strengthened, and the $11 \mu \mathrm{m} \mathrm{NH}_{3}$ absorption band is weakened (e.g., Leggett et al. 2007). Values of $\log K_{z z}=2-6$, corresponding to mixing timescales of $\sim 1 \mathrm{hr}$ to $\sim 10 \mathrm{yr}$ in the atmosphere, appear to be required to reproduce the observations of $\mathrm{T}$ dwarfs.

\subsection{8-4.1 $\mu \mathrm{m}$ Spectra}

Figure 4 shows the observed $0.8-4.1 \mu \mathrm{m}$ spectrum of HN Peg B compared to various model spectra. We treat the sedimentation and diffusion parameters $f_{\text {sed }}$ and $K_{z z}$ as independent parameters, although when full hydrodynamic models can be calculated, the grain sedimentation and replenishment will most likely be found to be intimately connected with the vertical transport of gas through the atmosphere. Here we use models with $f_{\text {sed }}=1,2,3,3.5$, and 4, and with $K_{z z}=0$ (equilibrium), $10^{2}, 10^{4}$, and $10^{6}\left(\mathrm{~cm}^{2} \mathrm{~s}^{-1}\right)$, and values of $T_{\mathrm{eff}} / \log g$ for each end of the allowed age range, 1015/ 4.22 and $1115 / 4.81(\S 5)$.

In all cases we find the spectra generated by the hotter temperature and higher gravity models do a better job of reproducing the data than the lower temperature and gravity models. Thus the age of the system appears to be at the older end of the allowed range, close to $0.5 \mathrm{Gyr}$. The best-fitting model has $T_{\text {eff }}=1115 \mathrm{~K}$, $\log g=4.81, f_{\text {sed }}=3.5$, and $K_{z z}=10^{4} \mathrm{~cm}^{2} \mathrm{~s}^{-1}$ (red line in Fig. 4). The cloud decks in this dwarf must therefore be relatively thin. Figure 4 also shows that significant vertical mixing in the atmosphere is required to reproduce the observed depth of the $3 \mu \mathrm{m}$ $\mathrm{CH}_{4}$ feature.

\subsection{4-8 $\mu \mathrm{m}$ Photometry}

Figure 5 compares the observed IRAC absolute magnitudes of HN Peg B to those calculated by our models. The models match the data quite well, except for the $4.49 \mu \mathrm{m}$ band, where the discrepancy is $\sim 0.4 \mathrm{mag}$. This is puzzling, given how well the model reproduces the $3-4 \mu \mathrm{m}$ spectrum (Fig. 4) and the $5.73 \mu \mathrm{m}$ magnitude. At these wavelengths the flux emerges from above the condensate clouds, and so our modeling of the cloud decks 
is not the source of the error. The problem either lies in the temperature-pressure profile - the region around the $0.5-1.0 \mathrm{bar}$ appears to be too hot - or in an opacity source that is missing or too weak in the models.

Nevertheless, the modeled photometry reproduces the data quite well, and supports the conclusions drawn from the comparison to the shorter wavelength spectrum-high sedimentation, thinner cloud decks are required, as well as significant vertical mixing. The best-fitting model for the $0.8-4 \mu \mathrm{m}$ spectrum is also the best choice to fit the IRAC photometry: $T_{\text {eff }}=1115 \mathrm{~K}, \log g=$ $4.81, f_{\mathrm{sed}}=3.5$, and $K_{z z}=10^{4} \mathrm{~cm}^{2} \mathrm{~s}^{-1}$.

\section{CONCLUSIONS}

The discovery by L07 of an early-T dwarf companion to a solar-like star offers an important test bed for models of the complex and poorly understood transition from the dusty L dwarfs to the clear T dwarfs. The new imaging data presented here improve the determination of the proper motion of this $\mathrm{T}$ dwarf, and confirm that the brown dwarf has common proper motion with the star, and hence is indeed a companion.

Our new high spatial resolution imaging data, together with the model comparisons to the $0.8-4.1 \mu \mathrm{m}$ spectra, show that the dwarf is most likely single, although it is possible that a much fainter, undetected, $<10 M_{\text {Jupiter }}$ brown dwarf lies within $100 \mathrm{AU}$ of the early-T dwarf.

Comparison of the observed and synthetic spectra generated by our models indicates that the HN Peg system is at the older end of the allowed age range, with an age $\sim 0.5 \mathrm{Gyr}$. Together with the measured luminosity, this implies that HN Peg B has $T_{\text {eff }}=1115 \mathrm{~K}, \log g=4.8$, radius $0.10 R_{\odot}$, and mass $28 M_{\text {Jupiter }}$.

Comparison of the observed and synthetic $0.8-8.0 \mu \mathrm{m}$ photometry and spectra also indicates that the condensate cloud decks in the photosphere of this $\mathrm{T}$ dwarf must be relatively thin, as we determine $f_{\text {sed }}=3.5$. Vertical mixing appears to be significant, as implied by the relative weakness of the $3 \mu \mathrm{m} \mathrm{CH}_{4}$ absorption band, which we fit with $K_{z z}=10^{4} \mathrm{~cm}^{2} \mathrm{~s}^{-1}$. In the future, objects like this, with well-determined age, radius, mass, gravity, and temperature, should allow development and testing of dy- namical atmosphere models that use a more physical approach to treat clouds and mixing. In the longer term, such models should provide the solution to the puzzle of the $\mathrm{L}$ to $\mathrm{T}$ dwarf transition.

This work uses observations obtained at the Gemini Observatory through Program GN-2007B-Q-22. The Gemini Observatory is operated by the Association of Universities for Research in Astronomy, Inc., under a cooperative agreement with the NSF on behalf of the Gemini partnership: the National Science Foundation (United States), the Science and Technology Facilities Council (United Kingdom), the National Research Council (Canada), CONICYT (Chile), the Australian Research Council (Australia), $\mathrm{CNPq}$ (Brazil), and CONICET (Argentina). We are very grateful to John Rayner for the NSFCAM2 observations. We would also like to thank the observers and queue coordinators who carried service observations at CFHT (programs 06BD94, 07AD98, and 07AD84). We gratefully acknowledge the Keck LGS AO team for their exceptional efforts in bringing the LGS AO system to fruition. It is a pleasure to thank Randy Campbell, Jim Lyke, Cindy Wilburn, Joel Aycock, and the Keck Observatory staff for assistance with the observations. This work is based and supported (in part) on observations made with the Spitzer Space Telescope, which is operated by the Jet Propulsion Laboratory, California Institute of Technology, under a contract with NASA. This publication makes use of data from the Two Micron All Sky Survey, which is a joint project of the University of Massachusetts and the Infrared Processing and Analysis Center, and funded by the National Aeronautics and Space Administration and the National Science Foundation, the SIMBAD database, operated at CDS, Strasbourg, France, and NASA's Astrophysics Data System Bibliographic Services. S. K. L.'s research is supported by the Gemini Observatory. M. C. L. and K. L. A. acknowledge support for this work from NSF grants AST 04-07441 and AST 05-07833, and an Alfred P. Sloan Research Fellowship.

Facilities: CFHT, Gemini:Gillett (NIRI), IRTF (SpeX), Keck:II
Ackerman, A. S., \& Marley, M. S. 2001, ApJ, 556, 872

Baraffe, I., Chabrier, G., Barman, T. S., Allard, F., \& Hauschildt, P. H. 2003, A\&A, 402, 701

Barnes, S. A. 2007, ApJ, 669, 1167

Bertin, E. 2006, in ASP Conf. Ser. 351, Astronomical Data Analysis Software and Systems XV, ed. C. Gabriel et al. (San Francisco: ASP), 112

Bertin, E., \& Arnouts, S. 1996, A\&AS, 117, 393

Blanco, C., Catalano, S., \& Marilli, E. 1979, A\&AS, 36, 297

Burgasser, A. J., Geballe, T. R., Leggett, S. K., Kirkpatrick, J. D., \& Golimowski, D. A. 2006a, ApJ, 637, 1067

Burgasser, A. J., Kirkpatrick, J. D., Cruz, K. L., Reid, I. N., Leggett, S. K., Liebert, J., Burrows, A., \& Brown, M. E. 2006b, ApJS, 166, 585

Burgasser, A. J., Marley, M. S., Ackerman, A. S., Saumon, D., Lodders, K., Dahn, C. C., Harris, H. C., \& Kirkpatrick, J. D. 2002, ApJ, 571, L151

Burrows, A., et al. 1997, ApJ, 491, 856

Cushing, M. C., Rayner, J. T., \& Vacca, W. D. 2005, ApJ, 623, 1115

Cushing, M. C., et al. 2006, ApJ, 648, 614 2008, ApJ, 678, 1372

Dahn, C. C., et al. 2002, AJ, 124, 1170

Fazio, G. G., et al. 2004, ApJS, 154, 10

Fegley, B., Jr., \& Lodders, K. 1996, ApJ, 472, L37

Fischer, D. A., \& Valenti, J. 2005, ApJ, 622, 1102

Geballe, T. R., Saumon, D., Leggett, S. K., Knapp, G. R., Marley, M. S., \& Lodders, K. 2001, ApJ, 556, 373

Golimowski, D. A., et al. 2004, AJ, 127, 3516

Hodapp, K. W., et al. 2003, PASP, 115, 1388

Hubeny, I., \& Burrows, A. 2007, ApJ, 669, 1248

Knapp, G. R., et al. 2004, AJ, 127, 3553

\section{REFERENCES}

König, B., Guenther, E. W., Esposito, M., \& Hatzes, A. 2006, MNRAS, 365, 1050 Kraus, A. L., Ireland, M. J., Martinache, F., \& Lloyd, J. P. 2008, ApJ, 679, 762 Leggett, S. K., Saumon, D., Marley, M. S., Geballe, T. R., Golimowski, D. A., Stephens, D. S., \& Fan, X. 2007, ApJ, 655, 1079

Liu, M. C., Leggett, S. K., \& Chiu, K. 2007, ApJ, 660, 1507

Liu, M. C., Leggett, S. K., Golimowski, D. A., Chiu, K., Fan, X., Geballe, T. R., Schneider, D. P., \& Brinkmann, J. 2006, ApJ, 647, 1393

Luhman, K. L., et al. 2007, ApJ, 654, 570 (L07)

Marley, M. S., Saumon, D., Guillot, T., Freedman, R. S., Hubbard, W. B., Burrows, A., \& Lunine, J. I. 1996, Science, 272, 1919

Marley, M. S., Seager, S., Saumon, D., Lodders, K., Ackerman, A. S., Freedman, R. S., \& Fan, X. 2002, ApJ, 568, 335

Metchev, S., \& Hillenbrand, L. A. 2006, ApJ, 651, 1166

Metchev, S., Kirkpatrick, J. D., Berriman, G. B., \& Looper, D. 2008, ApJ, 676, 1281

Monet, D. G., et al. 2003, AJ, 125, 984

Mugrauer, M., Seifahrt, A., Neuhäuser, R., \& Mazeh, T. 2006, MNRAS, 373, L31 Perryman, M. A. C., et al. 1997, A\&A, 323, L49

Pravdo, S. H., Shaklan, S. B., Wiktorowicz, S. J., Kulkarni, S., Lloyd, J. P., Martinache, F., Tuthill, P. G., \& Ireland, M. J. 2006, ApJ, 649, 389

Puget, P., et al. 2004, Proc. SPIE, 5492, 978

Roeser, S., \& Bastian, U. 1988, A\&AS, 74, 449

Saumon, D., Geballe, T. R., Leggett, S. K., Marley, M. S., Freedman, R. S., Lodders, K., Fegley, B., Jr., \& Sengupta, S. K. 2000, ApJ, 541, 374

Saumon, D., Marley, M. S., Cushing, M. C., Leggett, S. K., Roellig, T. L., Lodders, K., \& Freedman, R. S. 2006a, ApJ, 647, 552

Saumon, D., Marley, M. S., Lodders, K., \& Freedman, R. S. 2003, in IAU Symp. 211, Brown Dwarfs, ed. E. L. Martín (San Francisco: ASP), 345 
Saumon, D., et al. 2006b, ApJ, 647, 552

Shure, M. A., Toomey, D. W., Rayner, J. T., Onaka, P. M., \& Denault, A. J. 1994, Proc. SPIE, 2198, 614

Simons, D. A., \& Tokunaga, A. 2002, PASP, 114, 169

Skrutskie, M. F., et al. 2006, AJ, 131, 1163

Tokunaga, A. T., Simons, D. A., \& Vacca, W. D. 2002, PASP, 114, 180
Trilling, D. E., et al. 2008, ApJ, 674, 1086

Valenti, J. A., \& Fischer, D. A. 2005, ApJS, 159, 141

van Dam, M. A., et al. 2006, PASP, 118, 310

Werner, M. W., et al. 2004, ApJS, 154, 1

Wizinowich, P. L., et al. 2006, PASP, 118, 297 